\title{
Design of University MOOC Education Information Management System
}

\author{
Xiao Yu \\ School of Information Engineering ,Wuhan Technology and Business University, Wuhan, P. R. \\ China,430065
}

123189990@qq.com

Keywords: Information management system; MOOC; Educational information system; University education ; JSP

\begin{abstract}
This paper analyzes the necessity of MOOC education information management system in the design of university MOOC education information. Through the development of MVC design pattern based on Web application, the system function module is analyzed, the overall architecture of the system is designed, and the database is accessed by JDBC. The user management, video management and post management module are realized. The system can manage the video information in MOOC education, set up user access rights, and has good practicability and expansibility.
\end{abstract}

\section{Introduction}

Education has been the focus of attention from all walks of life in the Internet today, the way of education has become more diversified, University MOOC education information management system is now in the context of the rapid development of the Internet, the emerging of an educational platform. MOOC provides a possibility for the public to accept high quality education and become an important way to expand the democratization of education, and also become one of the important means to enhance the influence of colleges and universities [1].As MOOC majority of course construction began in the computer professional, and thus the computer and software professional teaching methods and learning the greatest impact [2].MOOC rise can not be separated from the network teaching system support [3]. With the emergence of emerging technology innovation model of distance education, MOOC platform as the main medium of open and open education, to assist teachers to carry out teaching activities and to achieve teaching interaction provides a strong support [4].

University MOOC education information management system is the ideal classroom to reproduce, so that students can learn anytime, anywhere, self-learning, the fragmented time to be used. The system is based on the convenience of students learning, diversity design.

\section{Development System Background}

Introduction to MOOC.MOOC is a large-scale online course, is open, the English called Massive Open Online Courses. MOOC offers a large number of free courses, so that a large number of excellent educational resources to all over, to enhance the level of education, and even expand contacts. The emergence of MOOC, so that excellent educational resources to be shared. Internet open class is the reproduction of the traditional curriculum, compared with the MOOC, it is not attractive, not targeted, student interaction is low. MOOC is the presentation of the ideal classroom, there is a sense of learning scene, and the entire learning cycle can be all on the network to be presented, fully consistent with teaching and learning of personalized, scientific. The current MOOC system [5 - 8], in addition to providing tuition certificates for learners, there is no other systematic learning incentive, which is not conducive to learners complete course learning. MOOC 'ground to root' the best way is for students on campus using mixed learning model [9], MOOC course to provide students with a specific knowledge of the video to explain and conduct real-time test feedback, so as to improve the interaction between students and learning objects, to facilitate students to master the progress and effect of learning [10].MOOC education platform is mainly on the excellent educational resources on the network, 
sharing of educational resources. Teachers upload video, upload operations. Students watch the video anytime, anywhere, finish the job and comment.

University MOOC Education Information Management System Development of the Necessity. With the popularity of the Internet, the network has penetrated into all aspects of life. University MOOC education information management system is in the network coverage under the premise of the development of education platform, compared with the network open class, it is more user-friendly, interactive and scientific. MOOC is the presentation of the ideal course, the entire semester cycle of all the content on the network to be presented. Therefore, through the University MOOC education information management system, students can learn from their own online, after the completion of the homework to the teacher after the correction, the question of doubt in the discussion area with the students and teachers to discuss, and finally through the examination to detect themselves. It not only shared the quality of educational resources, but also to stimulate students' interest in learning.

This article is the university MOOC education information management system, through the university MOOC education information management system understanding, can publish quality education resources, allowing users to focus on courses, self-learning. Users can freely learn the progress of learning, learning anytime, anywhere, participate in interactive activities, to participate in the discussion. It is learner-centered, so that learners are willing to learn, take the initiative to learn.

\section{Development Technology}

The Web application of this system adopts MVC design model, which combines the technologies of JSP, Servlet and JavaBean, and divides the input, output and processing flow of Web application into three layers according to the view, model and controller. Among them, the view is mainly used to interact with the user, the system interface display is a view. The model is mainly used for business processing, the system login, registration, modify, delete and other functions is achieved by the model part. The control layer mainly responds to the user's request. The layers are independent and interrelated and work together.

Introduction to Eclipse and MyEclipse. Eclipse is a Java-based cross-platform free integration development environment, it is an open source development platform. Eclipse is a collection of frameworks and a set of services, building the development environment is mainly through the plug-in components, and it also comes with a standard set of plug-ins. Eclipse was originally used for Java language development, but through plug-ins, it can also be used as $\mathrm{C}++$ and Python language development tools.

MyEclipse is an extension to the Eclipse development environment and is an environment that supports J2EE integration development. It supports configuration, code writing, debugging and testing, and supports JavaScript, HTML, Struts, CSS, JSP, SQL, Hibernate and other practical needs, and J2EE and database development, publishing, integration and so on to improve work efficiency. As MyEclipse's powerful features, MyEclipse by the developers of all ages. The system used by the development environment is more powerful MyEclipse, the version is MyEclipse8.6.

Introduction to Navicat For MySQL. Navicat For MySQL is a powerful tool, mainly for database management and development, is designed for MySQL. It is used in a wide range, can be used for 3.21 or above version of the MySQL database server, and can be very support for most of the latest version of MySQL features. It uses a very good graphical user interface, effectively reducing the development costs and improve the developer's development efficiency.

Introduction to JSP. Dynamic web technology JSP, which is the basis of Java Web development, running the program from the beginning of it. JSP technology is based on Servlet, JSP interface is dynamic, the user through the interface to display the information, you can enter the information request in the interface. JSP can also be seen as a scripting language, in the traditional HTML file to insert Java programs and JSP tags, the formation of a JSP file.

Introduction to Tomcat. Tomcat is one of the most popular Web application server, it has a simple, scalable, and the advantages of small resources, and support all JSP and Servlet specifications, so the system development and testing are often used. Tomcat is part of the expansion of the Apache server, 
but the ability to deal with static HTML Apache server is not good, Tomcat configuration is completed, Tomcat is running JSP pages and Servlet business processing. Tomcat is a lightweight application server, very suitable for beginners to learn, but also suitable for use in small and medium-sized systems. Tomcat version of the many, the system is using Tomcat 6.0.37.

\section{System Design}

Functional Module Analysis. The home page is the core page of the system, any user needs to browse the home page before allowing to browse other pages, regardless of the user page is the current page, you can quickly return to the home page. Users can first browse the interface information, click on the course information, you can easily watch the course, in the viewing process, you can also post. Users click on the post information, you can view the post, and in the login conditions to reply to the post, the user can also post in their own post. If the user is a teacher, the teacher can upload the course and delete the course in his / her own course. If the login user is an administrator, click into the background, you can enter the background interface to manage the system. The system function block diagram is designed, as shown in Fig .1.

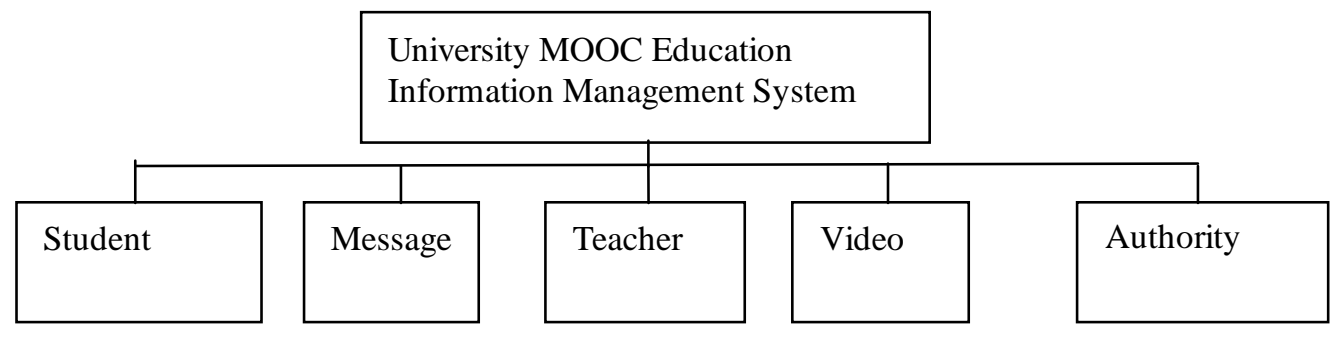

Figure 1. System Function Block Diagram

Overall Architecture Design. University MOOC education information management system is divided into four layers, described below.

View Layer. Mainly shows the user interface, user-friendly information or send a request, mainly using JSP technology to achieve.

Process Control Layer. Mainly used Servlet implementation, for business processing, data flow control.

Logical Processing Layer. Mainly using Java Bean to achieve, for the processing of data logic.

Public Class. Mainly used to store public needs of the class, such as data connection and other methods.

The main frame structure is designed. (refer with: Table 1)

Table 1 System Structure Table

\begin{tabular}{|l|l|l|}
\hline Level & $\begin{array}{l}\text { Program } \\
\text { name }\end{array}$ & Description \\
\hline Control & Java Code & $\begin{array}{l}\text { JDBCBean.javalbbsJava } \\
\text {.javal } \\
\text { Student.javalVideo.javal } \\
\text { Teacher.java }\end{array}$ \\
\hline View & Servlet & $\begin{array}{l}\text { bbsServletluserServlet। } \\
\text { videoServet }\end{array}$ \\
\hline Model & JSP & $\begin{array}{l}\text { Various types of JSP } \\
\text { programs }\end{array}$ \\
\hline
\end{tabular}

\section{Development Design and Implementation.}

Data Connection. This system is to use JDBC to access the database, for which the system specifically uses a class to store the database access information, when the need to access the data in the database, the use of the method, you can easily connect the database and shut down. 
Can be seen that there are two main methods, one used to connect to the database, one used to close the data connection. In the program, to use the database, just call these two methods can be, no need to write a link statement, to ensure the reusability of the code.

User Registration and Login. User registration is mainly for students and teachers, the administrator has a specified user name and password, so do not have to register the administrator. In the registration page, you can enter the student or teacher information, the default is the student registration. Click on the teacher to register, you can register the teacher information. After the user has filled in the basic information, submit the information, the system will enter the information into the control layer, according to the student and the teacher two different identities, the student information into the database student table, the teacher information into the database of teachers, and The user's password and other information into the database user table. In the user registration interface, the most important thing is how to allow students and teachers to register in the same interface. User login for students, teachers and administrators. When the user logs in, press the interface prompt to enter the login information.

Video Playback. Students, teachers and administrators can watch the video, and visitors who are not logged in can watch the video, but can not download and collect videos. Users in the home page of the video information list, select the video you want to watch, you can jump to the video playback interface. The system according to the click of the video, find the relevant video playback path, using Html5 video playback tags to achieve video playback.

Users can also click on the favorite video to enter the video playback interface. Video playback interface, the system according to the need to play the video information in the background to find the video storage path, the front desk to get the video storage path, according to the Video tab, the video broadcast out.

Posting and Replies. Users posting for students and teachers, users log on the system, you can view their posts, in the "My Posts" interface click release, you can jump to the post publishing interface. After submitting the post information, the system will pass the information into the control layer and store it in the database. Will be obtained from the front of the post information, insert into the post table, the post table in the issuer Id need to be based on the user name in the user table to find out.

Users can view their posts and other people's posts, in the post details interface, the user in the text box, enter the post reply message. After the user submits the information, it judges whether the user is logged in. If the user is logged in, the post reply information is saved in the database directly, otherwise it is anonymous reply.

Video Upload Download Delete Video. Video download, the user must first log on the system, that is not logged on the tourists can not download the video. The user in the video player interface, click the download button, the system to determine whether the user login, if not logged in, then jump to the login interface to $\log$ in, or to determine whether the file to download, if present, you can download the video to the local, Otherwise the prompt file does not exist. User video download, the system will be the information passed to the control layer, the system in the storage path, the file has been written to the local file. When the video file exists, the file will be written to the local file. When the file does not exist, the download fails.

After submitting the video information, the system will be the information obtained into the control layer, the video address and other related information into the database. The system will be submitted to the foreground of the information to resolve, if the general form information, the form information will be obtained out of the package. If the file information is uploaded, set the file storage path and write the file to the set path.

Teachers in the curriculum management interface to delete the video, click the delete button, the system will delete the video information to the background, the background to deal with video delete operation, change the video status to delete the state. Delete the video is not really the video information in the database completely deleted, but to modify the video information status. Video status 0 indicates no deletion, 1 means deletion. For deleted videos, the foreground will not be displayed and can not be played back, and the deleted video can be restored in the background. 
Student Management. Student management is part of the administrator operation, the administrator first log on the system, and then enter the background, in the background can be implemented on the management of student information. When the administrator selects the student information, the system automatically lists the unresolved student information, and the administrator can modify and delete the listed student information. Student identity changes need to first in the User table, the UserState state to teacher status, and then insert the student information into the teacher information table, and finally delete the student table information.

Teacher Management. Teacher management is part of the administrator operation, the administrator first log into the system, and then enter the background, in the background can be implemented on the management of teacher information. When the administrator selects the teacher information, the system automatically lists the undeleted information, and the administrator can modify and delete the listed information. The teacher changes the teacher's information according to the teacher, and changes the teacher's information.

Video Management. Video management is the administrator of the task, the administrator log on the system, you can enter the background, in the background to manage the video information. When the administrator selects the video information, the system automatically displays the undeleted information, and the administrator can modify and delete the listed information. After the user submits the video to delete the video, change the video status to the deleted status.

Message Management. Message management is the administrator of the task, the administrator log on the system, you can enter the background, in the background of the message information management. When the administrator selects the message information, the system automatically displays the undeleted information, and the administrator can modify and delete the listed information. After the user submits the message to delete the message, change the message status to the deleted state.

\section{Summary}

The system is based on the network system, so the use of Java Web development technology, and nowadays one of the most popular programming language to achieve the specific function of each module. System development using JSP technology, the front of the page using the HTML, JavaScript, Js, CSS, JQUERY and Bootstrap interface framework.

The basic functions of the system include the ability of students and teachers to log in to the system to view courses, publish and reply to posts. Teachers can upload courses, the administrator is mainly on the students, teachers, video, post management.

\section{References}

[1] Fan Wenqiang. Classification of large-scale network open course (MOOC) and its learning support based on relevanceism [J] .Chinese Journal of Distance Education, 2012 (3): 31-36.

[2] Xu Xiaofei. Grasp the opportunity of MOOC to promote the teaching reform of computer and software engineering [J]. China University, 2014 (1): 30-32.

[3] Han Xibin, Ge Wenxian, Zhou Qian, etc. MOOC platform and typical network teaching platform comparison [J]. China Audio-Visual Education, 2014 (1): 61-65.

[4] Zhang Hanyu, Mu Su, Ren Youqun. Comparison of Social Interaction Function of Learning Management System - Based on the Survey and Experience of Blackboard, Moodle and Sakai [J]. Modern Distance Education Research, 2013 (2): 38-42 .

[5] Adams S. Is Coursera the beginning of the end for the traditional higher education? [J]. Higher Education, 2012 (7): 12 - 13.

[6] Breslow L, Pritchard D E, De Boer J, et al. Studying in the worldwide classroom: Research into ed X 's first MOOC [J]. Research \& Practice in Assessment, 2013 (8): 13 - 25. 
[7] Chafkin M. Udacity's Sebastian Thrun, godfather of free online education, changes course [J]. Fast Company, 2013 (12): 14- 31.

[8] Sun Maosong, Xi Chunyan, Peng Yuanhong. A New Paradigm of MOOC Education in Flip Thinking [J]. Computer Education, 2014 (9): 1 - 2.

[9] Han Xibin, Zhai Wenfeng, Cheng Jiangang. c MOOC and x MOOC dialectical analysis and integration of higher education ecological chain [J]. Modern Distance Education Research, 2013, (6): 3-10.

[10] Sara Ibn El A. et.al. Massive Open Online Courses: A New Dawn for Higher Education? [J]. International Journal on Computer Science and Engineering,2013,5(5):323-323. 